\title{
GEOMETRIC CORRECTION FOR BRAILLE DOCUMENT IMAGES
}

\author{
Padmavathi. $S^{1}$,Aishwarya.A. $V^{2}$ and Iswaryah. $S^{3}$ \\ Amrita School of Engineering, \\ Amrita Vishwa Vidyapeetham, Coimbatore, India. \\ ${ }^{1}$ s_padmavathi@cb.amrita.edu, ${ }^{2}$ aishu.av7@gmail.com and \\ ${ }^{3}$ iswaryah8 8 @gmail. com
}

\begin{abstract}
Braille system has been used by the visually impaired people for reading.The shortage of Braille books has caused a need for conversion of Braille to text. This paper addresses the geometric correction of a Braille document images. Due to the standard measurement of the Braille cells, identification of Braille characters could be achieved by simple cell overlapping procedure. The standard measurement varies in a scaled document and fitting of the cells become difficult if the document is tilted. This paper proposes a line fitting algorithm for identifying the tilt (skew) angle. The horizontal and vertical scale factor is identified based on the ratio of distance between characters to the distance between dots. These are used in geometric transformation matrix for correction. Rotation correction is done prior to scale correction. This process aids in increased accuracy. The results for various Braille documents are tabulated.
\end{abstract}

\section{KEYWORDS}

Braille to text Conversion, Skew detection, skew correction

\section{INTRODUCTION}

Braille is a system of writing that uses patterns of raised dots to inscribe characters on paper. It therefore allows visually -impaired people to read and write using touch instead of vision. Its characters are six- dot cells, two wide by three tall. Any of the dots may be raised, giving 64 possible characters including 26 English alphabets, punctuations, numbers etc. Although Braille cells are used world-wide, the meaning of each cell depend onthe language that they are being used to depict.

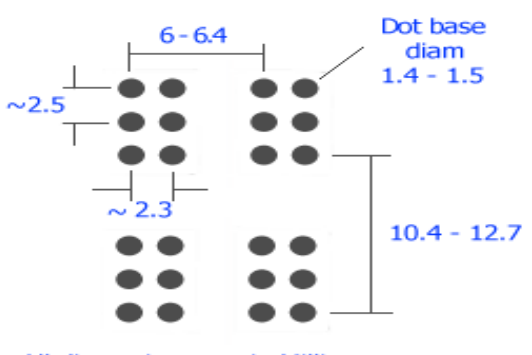

All dimensions are in Millimeters

Figure.1- Braille Cell Dimensions

David C. Wyld et al. (Eds) : CSITY, SIGPRO, AIFZ, NWCOM, DTMN, GRAPHHOC - 2016

pp. 35-46, 2016. ( ) CS \& IT-CSCP 2016

DOI : $10.5121 /$ csit.2016.60404 
The dimension of a Braille character is a standard one irrespective of the Language. Each dot in a Braille cell has a diameter of $1.5 \mathrm{~mm}$. Within each cell the dot centres are placed at a distance of $2.5 \mathrm{~mm}$ vertically and $2.3 \mathrm{~mm}$ horizontally. Two cells are separated by a distance of 6 to $6.4 \mathrm{~mm}$ horizontally and 10.4 to $12.7 \mathrm{~mm}$ vertically. These are illustrated in Figure.1.

Since the number of Braille books available are limited in number, when an ordinary text book is scanned and converted to text or audio, benefits many people who are in need. This also enables the corresponding document to be sent across the web. Identifying the Braille cells and decrypting them to the corresponding language are the major processes involved in the conversion[ 7]. Identifying Braille cells is difficult if the scanned documents are rotated or if those sheets are at different scale. These issues are briefed as follows

- When tilted to some angle, mapping of Braille to text is inappropriate and hence results in incorrect text.

- An inverted document may result in different mapping. An example of 2 Tamil letters, Inverted இis equivalent toஈ. Their Braille representations are in shown in Figure.2.

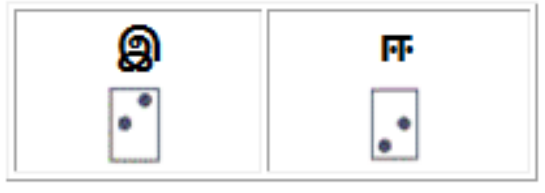

Figure 2.Inverted symbol mapping

- If the documents are either zoomed or shrunk their scaling varies and the spacing between the Braille dots or cells will be different from standard measurements.

Hence, the identification of Braille cells becomes difficult.

This paper proposes a technique which corrects the tilting of the Braille documents and brings the scanned document to the standard scale. The scanned document is converted to binary image before processing. The tilt or skew angle is identified by fitting a straight line to the set of first encountered vertical pixels. An inverse rotation matrix is used to correct the tilt. This paper utilizes the fact that ratio of the between character separation to within character separation should remain constant irrespective of scaling factor. A wide separation and a narrow separation horizontally signify the horizontal distance between two Braille cells (i.e. 2 characters and horizontal distance between the dots of the same Braille cell respectively. These distances cannot be calculated directly as the presence of dots varies for the characters present in the document. Since the dots raised may occur in different position, these distances varies for different character combinations. The ratio of distance between characters to distance between dots is between 2.6 to 2.78 in horizontal direction and 2.08 to 2.54 in vertical direction irrespective of the scaling factor. The distance pair satisfying these criteria is chosen and compared with the standard values to calculate the horizontal scale factor. A similar procedure is used for vertical distance and the vertical scale factor is calculated. An inverse geometric transformation matrix is used to bring the scale to the standard form. Once the documents are brought to the standard form the Braille characters could be converted to text as explained in [7].

In this paper, section 2 discusses about some of the methods proposed for finding rotation and 
scaling of scanned documents. Third section explains in detail about the solution proposed and fourth section covers the outcomes of the proposed method. Final section concludes with issues involved in implementing the proposed method and future works.

\section{LITERATURE REVIEW}

The issues that must be taken into consideration when implementing Braille to text conversion are scaling and rotation factors. Some of the methods to find them are as follows: Using the deviation over the sum of rows, [1] image was slanted over an angle. Each time it was slanted one pixel in vertical direction, deviation over sum of rows was calculated. When dots are horizontal, maximum was obtained. In [3], a probabilistic approach was proposed to estimate skewness and line-spacing of the Braille documents. Idea is to extract locations of the pixels belonging to shadow regions of dots and consider them as samples drawn from a twodimensional Probabilistic Density Function (PDF). Parameters of the PDF are related to skewness and scale properties of the document. These parameters are estimated in a Maximum-Likelihood framework using the Expectation Maximum approach. Paper [4] does the following for finding the rotation angle: Geometric correction aims to bring all scanned Braille document images to a common reference point. A simple technique is used to solve this issue: manually draw a $15 \times 10$ $\mathrm{mm}$ rectangle using black ink pen at a fixed position on each page of Braille document. By detecting the $15 \times 10 \mathrm{~mm}$ rectangle in the scanned image and estimating the position and orientation of the rectangle, transform the scanned image to a common reference point. Abdul Malik Al-Salman et al.[2] have proposed a probabilistic algorithm to correct any de-skewing in tilted scanned images. They also mention that maximum degree of recognizing a de-skewed image is 4 degrees from either the left or the right side.

As referred in [8] Supernova is a window-based magnifier, screen reader and a Braille system that supports the conversion of text to speech, Braille displays and note-takers. [9] refers to a paper on Braille word segmentation and transformation of Mandarin Braille to Chinese character. [10] discuss the main concepts related to OBR systems; list the work of different researchers with respect to the main areas of an OBR system, such as pre-processing, dot extraction, and classification. [11] focuses on developing a system to recognize an image of embossed Arabic Braille and then convert it to text. [12] proposes a software solution prototype to optically recognise single sided embossed Braille documents using a simple image processing algorithm and probabilistic neural network. [13] describes an approach to a prototype system that uses a commercially available flat-bed scanner to acquire a grey-scale image of a Braille document from which the characters are recognised and encoded for production or further processing. [14] presents an automatic system to recognize the Braille pages and convert the Braille documents into English/Chinese text for editing.[15] describes a new technique for recognizing Braille cells in Arabic single side Braille document. [16] developes a system that converts, within acceptable consrtraints , (Braille image) to a computer readable form. [17] provides a detailed description of a method for converting Braille as it is stored as characters in a computer into print.

\section{PROPOSED Solution}

Most of the commercial software focuses on converting text to Braille and vice versa. Very few consider scanned Braille documents as input and the geometric corrections are done based on 
probabilistic models. This paper proposes a method that uses a geometric transformation matrix for rotation and scale correction. The parameters of the matrix are identified using standard measurement of the Braille cells. The overall block diagram for the method proposed is shown in Figure 3.

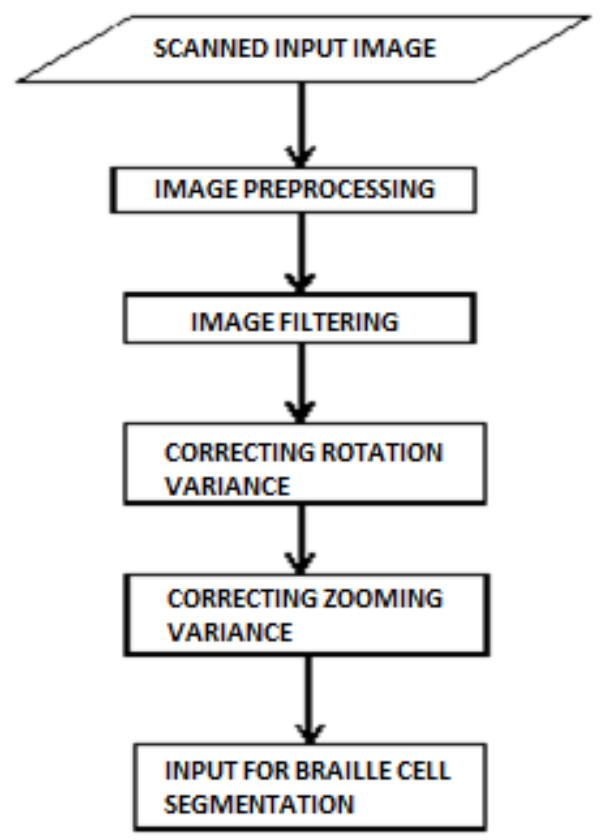

Figure.3. Block diagram of proposed method

The Braille document is scanned and taken as input. The scanned document is converted to gray scale and the noises are removed using Gaussian filter. To identify the dots, the image is convolved with the Prewitt filter and thresholding the resulting image. The skew correction of the document is done in two steps. First the rotation is corrected which is followed by scale correction. The Braille dots are represented as white and non-dots are represented as black in the resulting binary image.

\subsection{Rotation Correction}

Since the document is scanned, the maximum tilt is assumed to be 4 degrees in clockwise or anticlockwise direction. The extreme coordinate of the white dots are used to identify the direction of the tilt.

Let $\mathrm{x}_{\min }$ and $\mathrm{x}_{\max }$ represent the minimum and maximum $\mathrm{x}$ coordinates of the white dots. Their corresponding $\mathrm{y}$ values are represented as $\mathrm{y}\left(\mathrm{x}_{\min }\right)$ and $\mathrm{y}\left(\mathrm{x}_{\max }\right)$ respectively. Then the conditions in $\mathrm{Eq}(1)$ and (2) specify the anticlockwise and clockwise rotation as illustrated in Figure.4. and Figure.5 respectively.

$$
\begin{aligned}
& \left(\mathrm{x}_{\min }\right)<\mathrm{y}\left(\mathrm{x}_{\max }\right) \\
& \mathrm{y}\left(\mathrm{x}_{\min }\right)>\mathrm{y}\left(\mathrm{x}_{\max }\right)
\end{aligned}
$$


If both conditions fail then the document is not rotated.In the rotated document the global $\mathrm{y}_{\min }$ is identified and vertical lines are drawn to the first encountered white dots from $y_{\min }$. The dots are chosen only if its $\mathrm{Y}$ value falls in the range $\left[\mathrm{y}_{\min }, \mathrm{T}\right]$, where $\mathrm{T}$ represents a threshold.

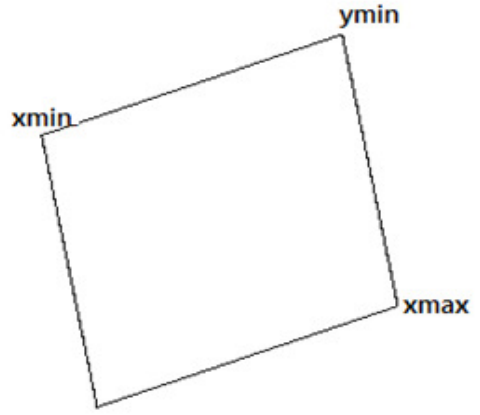

Figure.4. Sample image for anticlockwise rotation

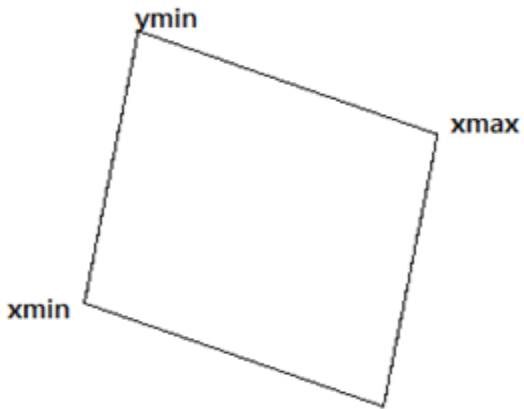

Figure.5. Sample image for clockwise rotation

A straight line is fit on the dots selected such that it minimizes the squared deviations of observed $\mathrm{Y}$ co-ordinates from any alternative line as in $\mathrm{Eq}(4)$.

$$
\begin{aligned}
& y_{i}^{\prime}=a+b x_{i} \\
& \sum e_{i}^{2}=\sum\left(y_{i}-y_{i}^{\prime}\right)^{2}
\end{aligned}
$$

Where $y_{i}$ is actual $y$ value, $x_{i}$ is independent variable $x$ value at $i^{\text {th }}$ position, $y_{i}^{\prime}$ is the predicted value of dependent variable, $a$ is a constant which representsthe point at which the line crosses the $\mathrm{Y}$ axis when $\mathrm{X}=0, \mathrm{~b}$ is regression slope, $\sum \mathrm{e}_{\mathrm{i}}^{2}$ is the sum of squared errors in prediction. The angle made by this line with the $\mathrm{X}$-axis is calculated and identified as the tilt angle ' $\alpha$ '. The sign of the tilt angle varies based on the direction of rotation. The rotation is corrected by applying the geometric transformation matrix as given in equation (5).

$$
\left[\begin{array}{l}
x \\
y \\
1
\end{array}\right]=\left[\begin{array}{lll}
\cos \alpha & \sin \alpha & 0 \\
-\sin \alpha & \cos \alpha & 0 \\
0 & 0 & 1
\end{array}\right] \times\left[\begin{array}{l}
x^{\prime} \\
y^{\prime} \\
1
\end{array}\right]
$$

\subsection{Scale Correction}

The tilt corrected image is used for scale correction to fit the Braille document to standard measurements. The binary image when projected on to $\mathrm{Y}$ axis gives the frequency of the white dots for each horizontal line. A zero frequency signifies the horizontal blank lines, which may occur between dots and between characters. A similar projection on $\mathrm{X}$ axis gives the frequency of white dots for each vertical line. A zero frequency here signifies the vertical blank lines that occur between dots and between characters. The presence of dots varies depending on the Braille character, hence the distance between the dots and characters also vary. To get a reasonable 
judgement of the distances, a sufficiently large sub-window near the top left corner is considered. This portion of the image is cropped such that the left and top blank lines are removed. The centroids of the dots in the cropped image are considered for further processing. The ratios of the horizontal distance between characters to distance between dots remain same irrespective of horizontal scaling factor. It ranges from 2.6 to 2.78. A similar concept is applicable for vertical distance ratio, which ranges from 2.08 to 2.54 . The horizontal distance between every successive pair of dot centroids are calculated. If the ratio of the distance falls in the specified range those distances are considered. Since the ratio falls in a range, the most frequently occurring value is found and its corresponding distance is used for calculating the scaling factor as shown in equation (6)

$$
S f=\frac{\text { ilstance of chosen cell }}{\text { standcrd cistance }}
$$

Where $S_{\mathrm{f}}$ is the scaling factor. The standard distance differs according to the dpi of the input image.For a 100 dpi image, pixel-mm equivalence could be found as follows. We know,

$$
\begin{aligned}
& 1 \mathrm{inch}=25.4 \mathrm{~mm} \\
& 100 \mathrm{pix} / \mathrm{inch}=100 \mathrm{pix} / 25.4 \mathrm{~mm} \\
& 1 \mathrm{~mm}=100 \mathrm{pix} / 25.4=3.93 \mathrm{pix}
\end{aligned}
$$

Hence from the Braille cell dimension the standard horizontal distance between characters varies from 23.58 to 25.15 pixels, the standard vertical distance between characters varies from 39.3 to 47.16 pixels. Once the scaling factor is known the image is de-scaled to standard size using scaling inverse transformation matrix in equation (10).

$$
\left[\begin{array}{l}
x \\
y \\
1
\end{array}\right]=\left[\begin{array}{lll}
1 / p & 0 & 0 \\
0 & 1 / q & 0 \\
0 & 0 & 1
\end{array}\right] \mathrm{x}\left[\begin{array}{c}
x^{\prime} \\
y^{\prime} \\
1
\end{array}\right]
$$

where $\mathrm{p}$ and $\mathrm{q}$ are the scaling factors obtained in horizontal and vertical direction from the input image respectively and $\left(\mathrm{x}^{\prime}, \mathrm{y}^{\prime}\right)$ are input pixel values and $(\mathrm{x}, \mathrm{y})$ are their corresponding output pixels.

Simple Braille cell overlapping could be done on the scale corrected image. The extracted cells can converted to Braille characters which can then be mapped to the corresponding alphabet as in [7].

\section{EXPERIMENTAL RESULTS}

Several Braille documents of different dpi's (say 100,300,400,600) were taken as input for both scaling and rotation correction. The result shown below refers to documents of $100 \mathrm{dpi}$. A portion of the scanned Braille document is shown in Figure.6. 


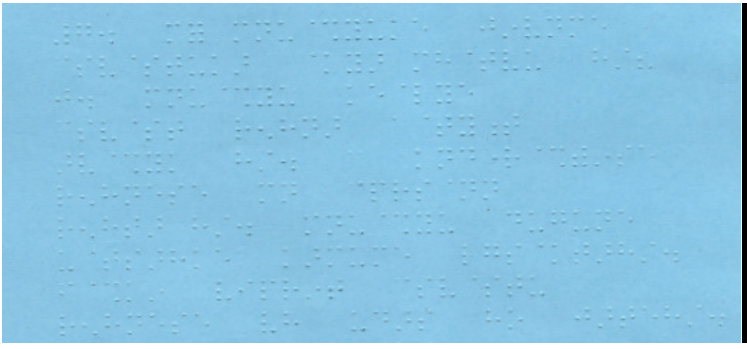

Figure.6. Scanned input image

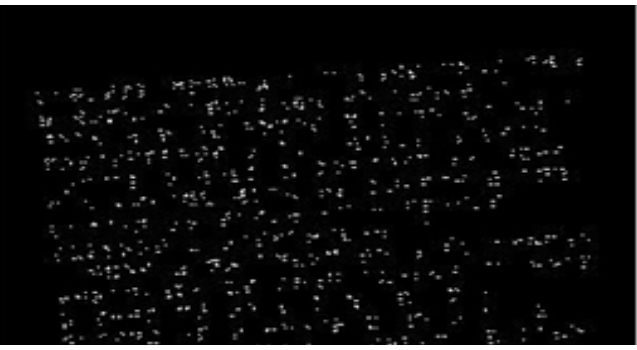

Figure.7. Binary image

The image is pre-processed and converted into binary image as discussed in previous section. A portion of the binary image is shown in Figure.7.

\subsection{Rotation}

The first occurring white dot when tracing vertically up to a threshold distance $\mathrm{T}$ is found. All such dots along the horizontal direction is indicated by vertical yellow lines in Figure.8. These dots are horizontally joined by the cyan line.

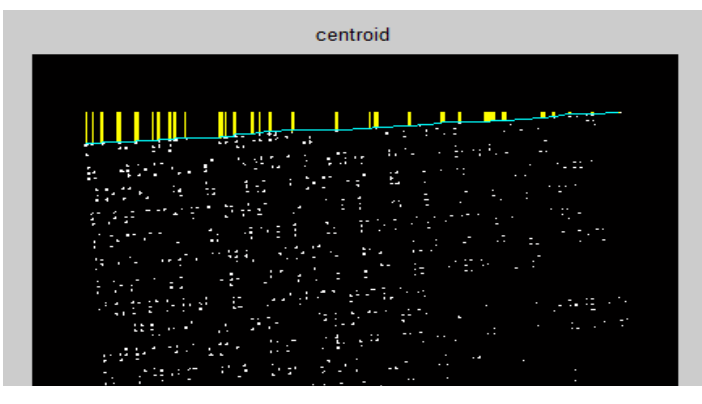

Figure.8 Before line fitting algorithm

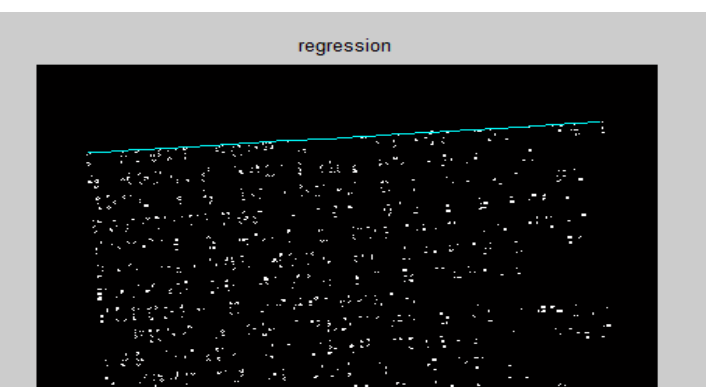

Figure.9. After line fitting algorithm

Image obtained after using line fitting algorithm for the dots which are lying in the cyan line in the Figure. 8 is shown in the Figure 9

The coordinates of dots so obtained are plotted as shown in Figure.10. It also shows the line with minimum error.

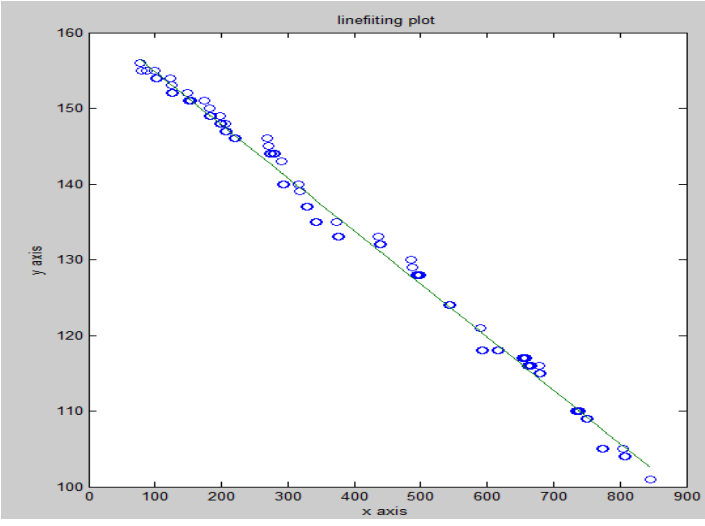

Figure.10 (a)graph for anticlockwise line fitting

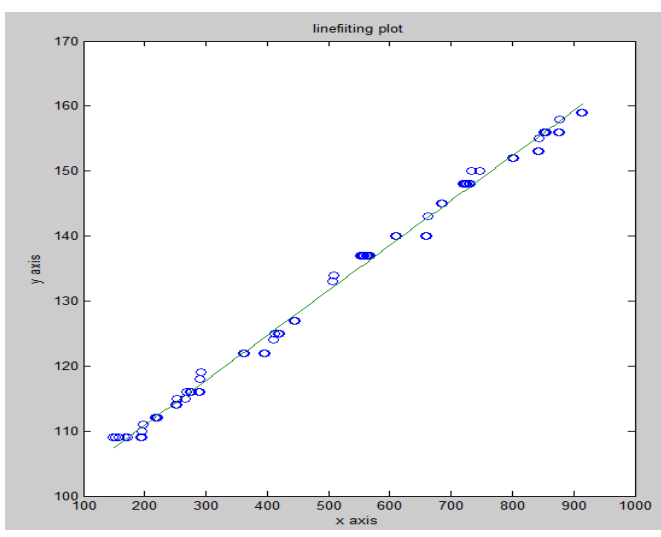

Figure.10(b)graph for clockwise line fitting 
The final image obtained after removing tilt is shown in Figure.11.

The actual rotation angle $(\theta)$ the starting point and end point before line fitting $\left(S_{b}\right.$ and $\left.E_{b}\right)$ and after line fitting $\left(\mathrm{S}_{1}\right.$ and $\left.\mathrm{E}_{\mathrm{l}}\right)$, the experimentally calculated angle $\theta_{\mathrm{c}}$ and mean error of line fitting $\mathrm{M}_{\mathrm{err}}$ are tabulated for different anticlockwise rotation angle in Table.1. A similar listing is done for clockwise rotation angle in Table.2.

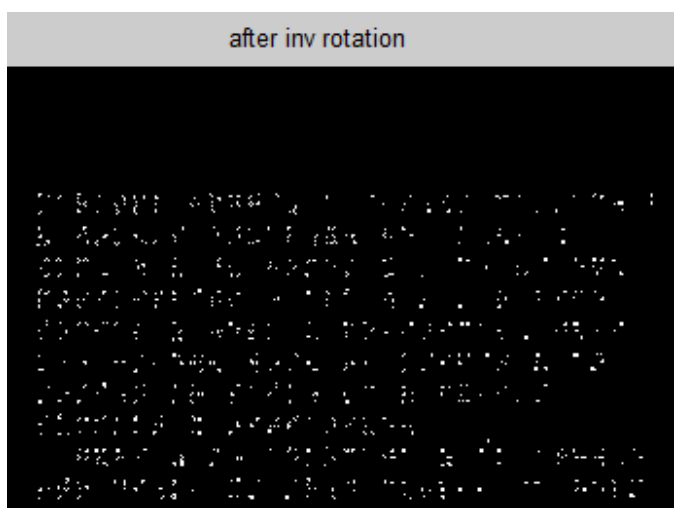

Figure.11. Re-rotated image

Table.1 anticlockwise rotation

\begin{tabular}{|l|l|l|l|l|}
\hline Actual $\theta$ & -4 & -3 & -2 & -1 \\
\hline Start point $\left(\mathrm{S}_{\mathrm{b}}\right)$ & $(79,155)$ & $(77,144)$ & $(79,137)$ & $(73,116)$ \\
\hline End point $\left(\mathrm{E}_{\mathrm{b}}\right)$ & $(845,101)$ & $(843,102)$ & $(850,107)$ & $(844,103)$ \\
\hline Start point $\left(\mathrm{S}_{\mathrm{l}}\right)$ & $(79,156)$ & $(77,143)$ & $(79,136)$ & $(73,115)$ \\
\hline End pt $\left(\mathrm{E}_{\mathrm{l}}\right)$ & $(845,103)$ & $(844,103)$ & $(850,107)$ & $(844,102)$ \\
\hline$\theta_{\mathrm{c}}$ & -4.01 & -2.99 & -2.177 & -0.97 \\
\hline $\mathrm{M}_{\text {err }}$ & -2.24 & -2.04 & 5.35 & -1.69 \\
\hline
\end{tabular}

Table. 2 clockwise rotation

\begin{tabular}{|l|l|l|l|l|}
\hline Actual $\theta$ & 4 & 3 & 2 & 1 \\
\hline Start point $\left(\mathrm{S}_{\mathrm{b}}\right)$ & $(915,159)$ & $(897,144)$ & $(883,136)$ & $(861,116)$ \\
\hline End point $\left(\mathrm{E}_{\mathrm{b}}\right)$ & $(149,109)$ & $(140,108)$ & $(126,110)$ & $(141,106)$ \\
\hline Start point $\left(\mathrm{S}_{\mathrm{l}}\right)$ & $(915,160)$ & $(897,145)$ & $(883,137)$ & $(861,116)$ \\
\hline End pt $\left(\mathrm{E}_{\mathrm{l}}\right)$ & $(149,107)$ & $(140,107)$ & $(126,109)$ & $(141,105)$ \\
\hline$\theta_{\mathrm{c}}$ & 3.95 & 2.89 & 2.09 & 0.91 \\
\hline $\mathrm{M}_{\text {err }}$ & -4.06 & 5.53 & -4.40 & 8.31 \\
\hline
\end{tabular}

\subsection{Scaling}

The image obtained as a result of rotation correction is given as input for scaling correction. Horizontal and vertical projections profiles are calculated. The horizontal and vertical lines with zero frequency after eliminating consecutive zeros are identified as Braille cell boundary and are shown in blue in Figure.12. 


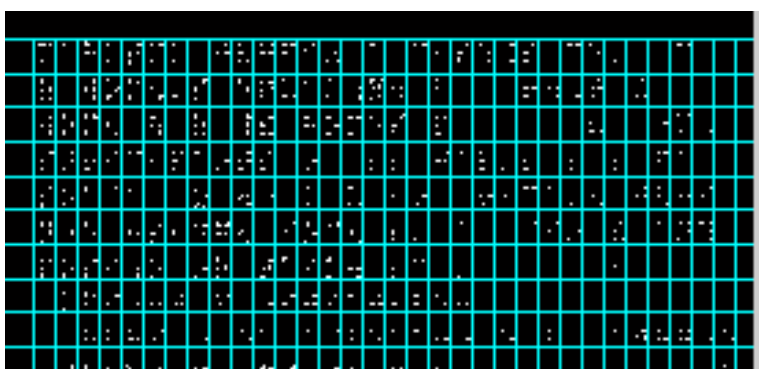

Figure.12 Image after Profiling

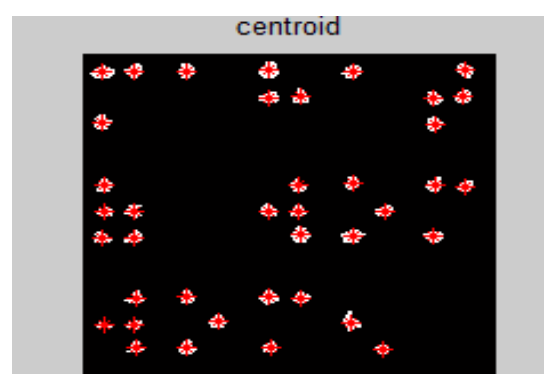

Figure.13. image with centroids marked

To decide the scaling factor and the ratio, 4 standard distances are considered. These distances are calculated with the dot centres as reference.

Case 1: distance in horizontal direction within the same cell $\left(\mathrm{w}_{\mathrm{h}}\right)$, which is $2.3 \mathrm{~mm}$ or 9.04 pixels for a standard Braille cell $\left(\right.$ Std $\left.\mathrm{w}_{\mathrm{h}}\right)$.

Case 2: distance in horizontal direction between 2 consecutive cells $\left(b_{h}\right)$. This distance according to standard measurement ranges from 6-6.4mm i.e. 23.58 to 25.15 pixels ( $\left.\operatorname{Std} b_{h}\right)$.

Case 3: distance in vertical direction within the same cell $\left(\mathrm{W}_{\mathrm{v}}\right)$, whose standard measurement is $2.5 \mathrm{~mm}$ or 9.82 pixels $\left(\mathrm{Std}_{\mathrm{v}}\right)$.

Case 4: distance in vertical direction between 2 consecutive Braille cells $\left(b_{v}\right)$. This distance according to standard measurement ranges 10.4 to $12.7 \mathrm{~mm}$ or 39.3 to 47.16 pixels $\left(S t d b_{v}\right)$.

Part of the profiled image is cropped and the centroids of each Braille dot are marked red in colour as shown in Figure. 13. From the cropped image the distances and ratios are calculated as specified in section 3 . The top 3 most frequently occurring ratios that fall within the valid range and their counts are tabulated in Table. 3 for various $\%$ of scaling. The horizontal distance values $\left(w_{h}\right.$ and $\left.b_{h}\right)$ for the most frequently occurring ratio is listed in Table.4. Factr1 and Factr2 represent the horizontal scale factor which are calculated from $\mathrm{w}_{\mathrm{h}}$ and $\mathrm{b}_{\mathrm{h}}$ respectively using the formula

$$
\begin{aligned}
& \text { Factr } 1=\mathrm{w}_{\mathrm{h}} \div \operatorname{Std}\left(\mathrm{w}_{\mathrm{h}}\right) \\
& \text { Factr } 2=\mathrm{b}_{\mathrm{h}} \div \operatorname{Std}\left(\mathrm{b}_{\mathrm{h}}\right)
\end{aligned}
$$

Table.3. Horizontal Ratio-count

\begin{tabular}{|l|l|l|l|l|l|l|}
\hline $\begin{array}{c}\text { size } \\
\%\end{array}$ & \multicolumn{3}{|c|}{ Ratio } & \multicolumn{3}{c|}{ Count } \\
\hline & r1 & r2 & r3 & c1 & c2 & c3 \\
\hline 25 & 2.60 & 2.64 & 2.69 & 6 & 3 & 2 \\
\hline 50 & 2.61 & 2.68 & 2.72 & 3 & 3 & 2 \\
\hline 75 & 2.66 & 2.70 & 2.60 & 5 & 2 & 1 \\
\hline Orig & 2.70 & 2.64 & 2.67 & 4 & 2 & 2 \\
\hline 125 & 2.64 & 2.75 & 2.68 & 4 & 2 & 1 \\
\hline 150 & 2.61 & 2.66 & 2.73 & 3 & 1 & 1 \\
\hline 175 & 2.60 & 2.73 & 2.76 & 3 & 2 & 1 \\
\hline
\end{tabular}


Table.4 Horizontal distance and scale factor

\begin{tabular}{|l|l|l|l|l|l|}
\hline Dist & $\begin{array}{l}\text { Within } \\
\text { char } \\
\left(\mathrm{w}_{\mathrm{h}}\right)\end{array}$ & $\begin{array}{l}\text { Betwn } \\
\text { char } \\
\left(\mathrm{b}_{\mathrm{h}}\right)\end{array}$ & $\begin{array}{l}\text { Ratio } \\
\mathrm{b}_{\mathrm{h}} \div \mathrm{w}_{\mathrm{h}}\end{array}$ & $\begin{array}{l}\text { Factr } \\
1\end{array}$ & $\begin{array}{l}\text { Factr } \\
2\end{array}$ \\
\hline 25 & 2.3 & 6 & 2.6 & 0.25 & 0.24 \\
\hline 50 & 4.67 & 12.2 & 2.61 & 0.51 & 0.49 \\
\hline 75 & 6.9 & 18.36 & 2.66 & 0.76 & 0.75 \\
\hline Orig & 9.04 & 24.47 & 2.7 & 1 & 1 \\
\hline 125 & 11.43 & 30.25 & 2.64 & 1.26 & 1.24 \\
\hline 150 & 13.56 & 35.45 & 2.61 & 1.5 & 1.45 \\
\hline 175 & 16.38 & 42.58 & 2.6 & 1.81 & 1.74 \\
\hline
\end{tabular}

A similar procedure is adopted for vertical distances. The valid vertical ratios range from 2.08 to 2.54. Factr3 and Factr4 in the Table.6 represent the vertical scale factors, calculated from $\mathrm{w}_{\mathrm{v}}$ and $b_{v}$ respectively using the formula

$$
\begin{aligned}
& \text { Factr3 }=w_{v} \div \operatorname{Std}\left(w_{v}\right) \\
& \text { Factr } 4=b_{v} \div \operatorname{Std}\left(b_{v}\right)
\end{aligned}
$$

Table.5 Vertical Ratio-count

\begin{tabular}{|l|l|l|l|l|l|l|}
\hline $\begin{array}{c}\text { size } \\
\%\end{array}$ & \multicolumn{3}{|c|}{ Ratio } & \multicolumn{3}{c|}{ Count } \\
\hline & r1 & r2 & r3 & c1 & c2 & c3 \\
\hline 25 & 2.16 & 2.41 & 2.34 & 4 & 2 & 2 \\
\hline 50 & 2.19 & 2.22 & 2.10 & 3 & 2 & 1 \\
\hline 75 & 2.21 & 2.32 & 2.25 & 4 & 2 & 1 \\
\hline Orig & 2.24 & 2.43 & 2.35 & 3 & 2 & 2 \\
\hline 125 & 2.25 & 2.15 & 2.41 & 4 & 2 & 1 \\
\hline 150 & 2.26 & 2.12 & 2.38 & 3 & 1 & 1 \\
\hline 175 & 2.24 & 2.36 & 2.17 & 3 & 2 & 1 \\
\hline
\end{tabular}

Table.6 Vertical distance and scale factor

\begin{tabular}{|l|l|l|l|l|l|}
\hline Dist & $\begin{array}{l}\text { Withi } \\
\mathrm{n} \text { char } \\
\left(\mathrm{w}_{\mathrm{v}}\right)\end{array}$ & $\begin{array}{l}\text { Between } \\
\text { char } \\
\left(\mathrm{b}_{\mathrm{v}}\right)\end{array}$ & $\begin{array}{l}\text { Ratio } \\
\left(\mathrm{b}_{\mathrm{v}} \div \mathrm{w}_{\mathrm{v}}\right)\end{array}$ & $\begin{array}{l}\text { Fact } \\
\mathrm{r3}\end{array}$ & $\begin{array}{l}\text { Factr } \\
4\end{array}$ \\
\hline 25 & 4.61 & 10 & 2.16 & 0.26 & 0.25 \\
\hline 50 & 9.04 & 19.87 & 2.19 & 0.50 & 0.49 \\
\hline 75 & 13.48 & 29.9 & 2.21 & 0.76 & 0.74 \\
\hline Orig & 17.74 & 39.9 & 2.24 & 1.00 & 1.00 \\
\hline 125 & 22.18 & 49.8 & 2.25 & 1.25 & 1.24 \\
\hline 150 & 25.9 & 58.65 & 2.26 & 1.46 & 1.48 \\
\hline 175 & 31.04 & 69.8 & 2.24 & 1.75 & 1.74 \\
\hline
\end{tabular}

To show the calculated distance between the Braille cells in horizontal and vertical direction, the centroids of the dots are marked in yellow colour in (Figure.14). The distance between one 
Braille character to another which lie in the range of standard measurement in either direction are marked in yellow colour in the figure.

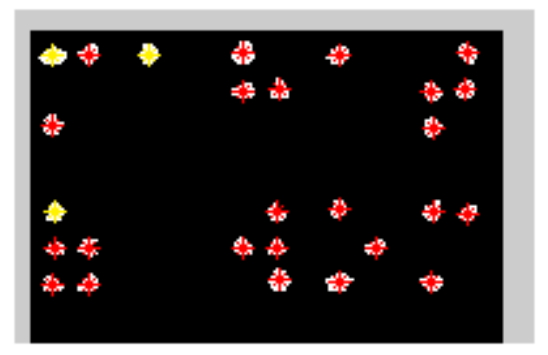

Figure.14. Horizontal and vertical distances

\section{CONCLUSION}

Extraction of text from the Braille document images requires skew detection and correction like normal document images. The skew detection for Braille documents is more challenging because it consists of dots only. This paper suggests a line fitting based skew correction method. The presence of noises could be mistaken for dots and hence noise removal and binarization process plays an important role in skew angle detection. A simple template matching could be used for Braille character extraction when the scale of the scanned document agrees with the standard measurement. The scale factor of the skew corrected image is calculated using the ratio of within character distance to between character distance.The arbitrary presence of dots is eliminated comparing with standard range and the maximum is calculated by voting system.

Braille documents when created manually, there is a possibility that the Braille dots may not be in a straight line, can be slanting between characters. This kind of skews could not be corrected by the method. An algorithm for skew correction for 180 degrees has to be modelled in future.

\section{REFERENCES}

[1] Jan Mennens, Luc van Tichelen, Guido Francois, and Jan J. Engelen (1994), “Optical Recognition of Braille Writing Using Standard Equipment”, IEEE Transactions on Rehabiliation Engg, Vol. 2, No. 4, December 1994.

[2] AbdulMalik Al-Salman, YosefAlOhali, Mohammed AlKanhal, and Abdullah AlRajih, "An Arabic Optical Braille Recognition System”, ICTA’07, April 12-14, Hammamet, Tunisia.

[3] MajidYoosefiBabadi, Behrooz Nasihatkon1, ZohrehAzimifar, Paul Fieguth, “ Probabilistic Estimation Of Braille Document Parameters", ICIP 2009.

[4] Jie Li and Xiaoguang Yan, Dayong Zhang, "Optical Braille Recognition with Haar Wavelet Features and Support-Vector Machine", 2010 International Conference on Computer, Mechatronics, Control and Electronic Engineering (CMCE).

[5] A. Antonacopoulos, D. Bridson, "A Robust BrailleRecognitionSystem" Proceedings of the IAPR International Workshop on Document Analysis Systems, Italy, 2004.

[6] J.A. Bilmes, "A Gentle Tutorial of the EM Algorithm and Its Application to Parameter Estimation for Gaussian Mixtureand Hidden Markov Models", Technical Report, University ofBerkeley,1998. 
[7] S.Padmavathi, Manojna K.S.S, Sphoorthy Reddy .S and Meenakshy.D, "Conversion Of Braille To Text In English, Hindi And Tamil Languages", in International Journal of Computer Science, Engineering and Applications (IJCSEA), Volume3 number 3 ,June 2013 pp19-32.

[8] AbdulMalik S. Al-Salman, "A Bi-directional Bi-Lingual Translation Braille-Text System", J. King Saud University, Vol. 20, Comp. \& Info. Sci., pp. 13-29,Riyadh(1428H./2008).

[9] Minghu Jiang etal, "Braille to print translations of Chinese",Information and Software Technology 44 (2002) 91-100

[10] Trends And Technologies In Optical Braille Recognition by AbdulMalik S. Al-Salman, Yosef AlOhali, and Layla O. Al-Abdulkarim, 3 'rd Int. Conf. on Information Technology,May 2007,Jordan.

[11] AbdulMalik Al-Salman, Yosef AlOhali, Mohammed AlKanhal, and Abdullah AlRajih,"An Arabic Optical Braille Recognition System",ICTA’07, April 12-14, Hammamet, Tunisia

[12] Lisa Wong,Waleed Abdulla,Stephan Hussmann,"A Software Algorithm Prototype for Optical Recognition of Embossed Braille", Pattern Recognition, 2004. ICPR 2004. Proceedings of the 17th International Conference on 23-26 Aug. 2004, 586- 589 Vol.2

[13] R.T. Ritchings, A. Antonacopoulos and D. Drakopoulos,"ANALYSIS OF SCANNED BRAILLE DOCUMENTS",In the book: Document Analysis Systems, A. Dengel and A.L. Spitz (eds.), World Scientific Publishing Co,1995, pp. 413-421

[14] C M Ng, Vincent Ng, Y Lau,"Regular Feature Extraction for Recognition of Braille", Computational Intelligence and Multimedia Applications, 1999. ICCIMA '99. Proceedings. Third International Conference, pages 302-306

[15] Zainab I. Authman, Zamen F.Jebr, "Arabic Braille scripts recognition and translation using image processing techniques", Journal: Journal of College of Education, Year: 2012 Volume: 2 Issue: 3 Pages: 18-26, Publisher: Thi-Qar University

[16] Jan mennues etal.,"Optical Recognition of Braille writing Using Standard Equipment", IEEE TRANSACTIONS ON REHABILITATION ENGINEERING, VOL. 2, NO. 4, DECEMBER 1994

[17] Paul Blenkhorn,"System For Converting Braille Into Print",IEEE TRANSACTIONS ON REHABILITATION ENGINEERING, VOL. 3, NO. 2, JUNE 1995 